'División de Cirugía, Programa de Estudios Médico Humanísticos, Facultad de Medicina, Pontificia Universidad Católica de Chile. Escuela de Psicología, Facultad de Ciencias Sociales, P. Universidad Católica de Chile. ${ }^{3}$ División de Cirugía, Facultad de Medicina, Pontificia Universidad Católica de Chile. Santiago, Chile. ${ }^{a}$ Cirujano Dentista. bPsicólogo, Magíster en Comunicación Social. Licenciado y candidato a Magíster en Filosofía, Universidad de Chile, estudiante de maestría en Estudios Literarios, Universidad de Buenos Aires, Argentina.

Recibido el 1 de octubre de 2012, aceptado el 18 de diciembre de

Correspondencia a: Hernán Ramírez Skinner, División de Cirugía, Facultad de Medicina, Pontificia Universidad Católica de Chile, Marcoleta 350, Santiago. Fono: 3543720 - 09-8258994 E-mail: hramirez.skinner@gmail.

\section{Dos mil años de separación entre medicina y cirugía: bases para comprender el fenómeno}

\author{
HERNÁN RAMÍREZ S. . $^{1, a}$, JERÓNIMO PARADA R. ${ }^{c}$, \\ CRISTIÁN CORTÉS S. ${ }^{2, b}$, SERGIO ZÚÑIGA R. ${ }^{3}$

\section{History of the split between medicine and surgery}

This paper summarizes historical and philosophical aspects, from Ancient Greece to the Middle Ages, concerning the split between physicians and surgeons that began to take place in Alexandria. When exploring the changes in classical medical thought and its influence on philosophy, we conclude that this historical moment was marked by a dual distinction between an essential being and a body subjected to becoming. From a unified mind in Hippocrates, in which medicine is based in knowledge of the relations, subjected to laws, of the organism exposed to natural forces $\Phi v \sigma \mathrm{i} \varsigma$ (physis among the Jonics), a rift amongst body and soul is conceived, specifically in Platonic thought, from which the surgical intervention of the body is considered improper of the medical art.

(Rev Med Chile 2013; 141: 402-406).

Key words: Education, medical; History of Medicine; History of Surgery.
$\mathrm{L}$ a cirugía ha sido una parte inherente del acto médico, desde los chamanes cavernarios hasta nuestros días. En Hipócrates vemos una mente unificada cuyas ideas fundamentan el

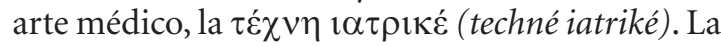
concepción unificada de alma y cuerpo en Hipócrates recibe la influencia de los físicos jónicos, a los que Giorgio Colli llama filósofos sobrehumanos $^{1}$. Sugiere llamar misticismo al movimiento de los presocráticos y señala al siglo VI a. C., como el momento en que interviene lo dionisíaco en forma decisiva en la vida espiritual de Grecia.

Tanto los presocráticos como Platón no separaban lo político de lo místico, porque la vida espiritual era unitaria y continua. Los griegos compartían su experiencia íntima para convencer a los hombres de comportarse políticamente en la vida de acuerdo con ella ${ }^{2}$. Esta experiencia la descubre en Anaximandro, Heráclito, Parménides y Empédocles, y sostiene que ellos alcanzan esta suprema "politicidad" sólo gracias a su interioridad dionisíaca y por eso los llama sobrehumanos.
Platón separa el mundo sensible, esto es, el mundo que deviene, del mundo suprasensible. Es aquí donde radicaría el inicio de una ruptura, una separación entre alma y cuerpo, ambos objetos del esfuerzo dietético del médico hipocrático para servir a la divina physis.

El presente artículo es descriptivo y contiene también algunos componentes analíticos o hermenéuticos, porque interpreta o sitúa el origen de la separación entre medicina y cirugía en un momento histórico determinado, a saber, en el pensamiento de Platón. Es decir, hacemos extensiva la separación que determina el pensamiento occidental por más de dos mil años, al ámbito médico.

\section{La medicina hipocrática como paideia}

Para Platón la auténtica paideia (paideia) es la formación del hombre para la educación $\alpha \rho \varepsilon \tau \eta ́ n$ (areté) total, para apoyar la acción del legislador. 
Éste es considerado como un educador que aspira infundir al estado un espíritu colectivo $\varepsilon$ ós (ethos) que da forma a todo ${ }^{3}$.

La educación es al mismo tiempo un asunto moral y práctico: honra a tus padres y aprende un oficio. La comunicación de conocimientos y habilidades profesionales es un conjunto transmisible y por lo tanto una $\tau \dot{\varepsilon} \chi \vee \eta$ (techné).

Jaeger considera a la medicina griega como paideia, porque el procedimiento de la medicina resumido por Hipócrates hace concebible a la ética socrática reflejada en los diálogos de Platón. Destaca la figura del médico demiurgo que trabaja con el gimnasta en la formación del cuerpo y con el músico, el poeta y el filósofo en la educación del espíritu.

Es la filosofía jónica de la naturaleza la que convierte a la medicina griega en un arte conciente y metódico. Anaximandro y Pitágoras sostienen lo que es adecuado a cada edad y sus fuerzas (teoría médica de la dieta). La reparación, una especie de compensación, se encuentra en la base de los procesos fisiológicos y patológicos y la $\_$$\sigma 0 \mathrm{o} \rho \alpha$ (isomoira) mantiene el estado de salud.

Anaximandro afirma como principio de la realidad a lo indefinido, lo indeterminado, lo infinito, a partir de lo que evoluciona todo lo real. Dado que nosotros sólo conocemos las formas particulares de materia que emanan de ese primer principio

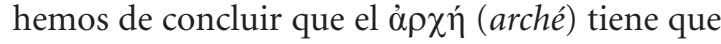
ser una materia desconocida para nosotros $y$, en cuanto tal, una materia indeterminada, indefinida, ilimitada, a la que Anaximandro da el nombre de

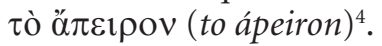

Cuando el maestro de Cos afirma que la enfermedad sagrada de la epilepsia no es ni más ni menos divina que cualquiera otra y responde a causas naturales igual que todas las demás, está afirmando la idea fundamental de la physis presocrática $^{5}$. La teoría de la naturaleza humana física traza el derrotero para todas las proyecciones del concepto sobre la naturaleza espiritual del hombre. Si el origen de las cosas es ápeiron, difícilmente Hipócrates reconocería algo sagrado en la epilepsia. Por el hecho de tener la enfermedad una causa natural, que se entronca ni más ni menos que con el origen de las cosas. En ese sentido, la physis es divina, no la epilepsia.

La techné que enseña la nueva ciencia médica se deslinda de la simple filosofía de la naturaleza y repudia a los médicos que exigen de un princi- pio armónico al que puedan reducirse todos los fenómenos concretos, como hacen los filósofos. Según Jaeger, el gran tema no estriba en lo que el hombre es, sino en "lo que es en relación con lo que come y bebe y a cómo vive y a los efectos que todo esto produce en él". En medicina deja de hablarse de la naturaleza humana como algo general. No hay una naturaleza, existen las naturalezas ${ }^{6}$.

La ciencia médica y la terapéutica del alma de Platón tienen en común que ambas derivan sus enseñanzas del conocimiento objetivo de la naturaleza misma. El médico se ocupa de la naturaleza del cuerpo, y el filósofo de la naturaleza del alma. El método hipocrático consiste en analizar la naturaleza, enumerar los tipos eĩos (eidos) y determinar lo adecuado para cada uno. Cuando dentro de una variedad se encuentra una unidad, se emplea el concepto de idea. Ambos conceptos metódicos, eidos e idea, los trasplanta Platón desde el cuerpo y sus funciones descritos por los médicos, a la ética y desde aquí a toda su ontología. Aristóteles se ayuda de la medicina y de una dieta sana, para resolver el problema de cómo el individuo ha de encontrar mediante el tacto seguro la verdadera pauta de su conducta, entre el exceso y el defecto en la regulación de los impulsos humanos, del placer y del dolor ${ }^{7}$.

La función del médico es la de un aliado de la naturaleza, los síntomas son un comienzo de la restauración del estado normal encauzado por el propio organismo. La naturaleza se ayuda a sí misma. La physis encuentra los medios y los caminos correctos sin necesidad de una inteligencia conciente y aún así, procede de un modo absolutamente teleológico. En una paideia inconciente, la naturaleza se encarga de hacer lo que es necesario ${ }^{8}$.

La separación platónica y la posición social que adquiere a partir de ella, le dio al médico griego una justificación para ejercer como sabio, filósofo, pedagogo de ricos y poderosos más que como práctico al lado del paciente, lo cual fue delegado posteriormente en los cirujanos ${ }^{9,10}$. De ahí el desprecio de los primeros sobre los segundos. Unos son médicos del alma que no tocan el cuerpo, los otros son prácticos, empíricos en el mejor de los casos, que atienden el sufrimiento del pueblo iletrado.

Los estados-ciudad de la Grecia del siglo IV a. C. probablemente organizaban la vida de los ciudadanos de manera que ellos pudieran disponer de tiempo para el cultivo del espíritu y el cuidado 
del cuerpo. Platón combina las tres virtudes físicas con las virtudes del alma, en una unidad armónica. La cultura física, tal como la conciben los médicos y gimnastas griegos, es también algo espiritual y, así, crean lo bueno y lo justo en todos los órdenes de la vida. El ideal helénico de la cultura humana era el ideal del hombre sano.

\section{La dualidad platónica en medicina}

Para Platón, fundador de la metafísica, lo inmutable como sustrato, nombra tanto la idea como dios, substancia, cosa en sí, espíritu absoluto, sujeto, voluntad como el en sí de las cosas, mónada, y todas las determinaciones que fundamentan el devenir desde el ser visto como lo siempre existente, ubicado en otro ámbito que el de la sensibilidad ${ }^{11}$. A través de la razón vemos el mundo y la razón se transforma así en nuestros ojos y a través de ellos aparece lo inmutable como fundamento del mundo del devenir, negando los sentidos que muestran el cambio ${ }^{12}$.

Nietzsche, desde el inicio de su camino pensante, ve a Platón como un síntoma de la decadencia griega, como el comienzo de la negación de la vida, en la medida que a través de la razón se fingen, por decirlo en sus términos, las ideas que están por encima del mundo del devenir y lo fundamentan ${ }^{13}$.

El saber $\dot{\varepsilon} \pi \imath \sigma \tau \mid \mu \eta$ (episteme) consiste en ver la idea, $y$ todo hablar que no tenga en vista a la idea en su decir es mera opinión $\delta o ́ \xi \alpha$ (doxa). Saber es ver lo inmutable suprasensible que constituye el ser de lo que es, ver la realidad eterna y, por lo tanto, la vida humana no le aparece como gran cosa al que es capaz de acceder a dicho conocimiento.

La vida terrenal aparece aquí como algo carente de valor en contraposición a las ideas, ellas mismas ordenadas por la idea del bien, idea suprema que

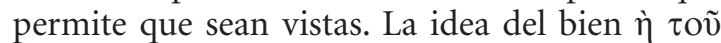
$\alpha \gamma \alpha \theta$ oṽ i̇́ź $\alpha$ (tou agathou idea) es lo fundante de todo cuanto es, el principio en tanto permite el conocimiento de las ideas y con ello de lo verdaderísimo, inaccesible por medio de los sentidos. A partir de la idea del bien se ordena el mundo, ella gobierna todo en la medida en que da la posibilidad del conocimiento, de la eterna verdad ${ }^{14}$.

Nietzsche ve aquí tanto la prefiguración del cristianismo, al que nombra como un "platonismo para el pueblo", como de todo el pensamiento metafísico posterior que se caracteriza por esta misma dualidad inaugurada en el pensamiento de Platón, que separa el mundo sensible, esto es el mundo que deviene, del mundo suprasensible ${ }^{15}$.

El Corpus hipocrático hunde sus raíces en la physis jónica y entrega el fruto de la medicina científica como paideia que apunta a cultivar en armonía las virtudes de cuerpo y espíritu. De esta unidad original de la mente griega del siglo $\mathrm{V}$ a. C. se pasa a una división de la praxis médica con la aparición de los cirujanos en Alejandría, en tiempos posteriores a Ptolomeo.

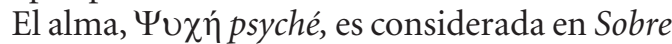
la dieta como una parte sutil e invisible del cuerpo, que se nutre y desarrolla a lo largo de la vida y cuyas funciones propias son el pensamiento, la conciencia y la afectividad. Pone el autor su interés en el orden natural más que en aquello que ordena, es decir, calla a propósito sobre la explicación del orden cósmico por acción de voũ $̧$ nous, la mente ${ }^{16}$. El alma se constituye por una proporción armónica entre partes insustituibles que le dan origen y expresión. Mediante la dieta es posible mejorar o deteriorar sus características naturales.

Para Anaxágoras "el hombre es un animal inteligente porque tiene manos", a lo cual replica Aristóteles diciendo que "el hombre tiene manos porque es un animal inteligente". Aquí, lo que en el jónico se encuentra unificado, en el estagirita simula la supremacía de lo espiritual sobre lo corporal.

El alma es la especificación viviente del principio que da forma a la materia, así lo plantean Platón y Aristóteles. Galeno reduce la sustancia del alma a la mezcla de las cuatro cualidades básicas y renuncia a ocuparse de lo incorpóreo dudando que exista algo no corpóreo en la naturaleza del hombre, heredando así la tradición de los presocráticos y constituyéndose en un filósofo de la naturaleza.

Tres fueron los métodos que empleó el médico hipocrático para servir fiel, cuidadosa y reverencialmente a la divina physis: la farmacoterapia, la dieta y la cirugía. A la tríada terapéutica original añadimos un cuarto elemento, la psicoterapia ${ }^{9,10}$. Desde las curaciones mágicas en los templos de Asclepio, del período mitológico de la medicina griega hasta la mancha del alma en la etiología galénica, los médicos procuraron tratar el cuerpo al mismo tiempo que el espíritu, dotando de la fuerza persuasiva del discurso a las intervenciones farmacológicas y quirúrgicas. El médico es demiurgo o artífice-operario del bien del pueblo por medio de una dietesis ejercida sobre el cuerpo 
e interpretada al mismo tiempo como instrumento de salud social y como medicina del alma.

Revisando el rol del médico en la sociedad vemos al médico hipocrático, heredero del saber jónico sobre la naturaleza, usando el empirismo y el método científico. Convive con el filósofo platónico dando su discurso doctoral, para quien lo suprasensible era el único espejo donde se refleja el ser y con los prácticos iletrados. En su trabajo en equipo democrático con gimnastas, poetas, músicos y filósofos, el médico griego encuentra una ética, un oficio basado en la técnica, transmisible como paideia, con la cual crea ciudad.

No tarda la asistencia médica en ser entregada a tres categorías de pacientes: los esclavos, los ciudadanos libres y pobres y los ciudadanos libres y ricos. Los esclavos son atendidos por los servidores de los médicos, los pobres reciben un tratamiento médico resolutivo y enérgico, y los ricos, sensibles al menor síntoma, prefieren tener un médico siempre vigilante frente a la enfermedad y la muerte.

El acto médico es un acto ético que apunta al enfermo, a la polis y a los compañeros del $\operatorname{arte}^{17}$. Al intervenir sobre el cuerpo y el espíritu del hombre, el médico griego contribuye a una sociedad de hombres sanos. A pesar de lo anterior, el médico griego cultivó una moral del éxito, sed de prestigio y una actitud competitiva, fenómeno del cual da testimonio Hipócrates en Sobre la Ciencia Médica.

El médico del helenismo alejandrino y romano ejerció poco después de la fundación de Alejandría en 331 a. C. hasta la caída del imperio bizantino en manos de los turcos otomanos en 1453. Se sintió ante la physis más un gobernador que un servidor. Junto con ello, concibió la $\pi \rho \bar{\alpha} \xi ı$ is praxis médica como un deber de filantropía hacia la humanidad, análogo al pensamiento cristiano sobre el prójimo. El sacramento de unción de los enfermos acompaña a los cuidados médicos y morales, la creación de la institución hospitalaria es una forma ética de caridad operativa ante los hechos de la enfermedad y el dolor, igualitaria para griegos y bárbaros, libres y esclavos, pobres y ricos.

Pronto, los padres cristianos elaborarán una teoría antropológica del pecado y de la penitencia, una teología moral, una antropología cristiana. La enfermedad ya no es el castigo de los dioses, ni la necesidad de la dinámica del cosmos, sino una prueba, a la manera de Job. La idea judeo-cristiana de la omnipotencia divina se impone a la filosofía jónica de la naturaleza.
Los médicos bizantinos posteriores a Galeno representaron la decadencia de la mente griega y la supremacía neoplatónica de un dios trascendente al mundo sobre una naturaleza penetrada por el logos. La clase dominante e ilustrada sufrió la presión teocrática y los estudios filosóficos y científiconaturales fueron cediendo terreno a los teológicos y jurídicos. Los ricos y poderosos fueron atendidos por médicos famosos como Oribasio y Aecio. Los pobres también accedían a cuidados médicos en términos de caridad y beneficencia, pero una asistencia médica socializada en derecho y justicia nunca llegó a existir. Las curas mágicas a la manera de la incubatio pero de corte cristiano, tuvieron una clientela nutrida entre el pueblo ignorante.

La profunda unidad de la cultura islámica medieval permitió preservar los clásicos, rescatándolos de las manos bárbaras, traduciéndolos, ilustrándolos y aportando contenidos originales.

En el siglo XI coexisten dos formas de enseñar la medicina en el mundo islámico. Están los defensores de un saber amplio en lógica, matemática, física y teología, imagen del médico completo ${ }^{18}$. Por otro lado, los partidarios del aprendizaje directo junto al paciente y bajo la tutoría del maestro. Nuevamente aparece la dualidad entre filósofos y prácticos, no obstante, los médicos combinaron ambos modelos de educación en diferentes proporciones ${ }^{19}$.

Durante la Edad Media el progreso en el arte de curar se debió a médicos profesionales y la enseñanza formal de la medicina se estableció en las recién creadas universidades de Salerno, Montpellier, París, Bolonia, Padua, Nápoles y otras.

Hacia el año 904 la escuela de Salerno estaba consolidada, sus publicaciones propias y copias de textos mostraban un concepto marcadamente empírico y un cierto desdén por la filosofía. En este ambiente prospera la práctica y la enseñanza de la cirugía.

La escuela de Montpellier retornó a la medicina basada en los clásicos incorporándolos en la enseñanza. Fundada en los siglos VIII-IX, se desarrolló entre los mundos latino y árabe con mediación intelectual de sabios hebreos.

Entre los años 1100 y 1400 se fundaron universidades en Europa y la de París, así como otras en Francia, Italia, Alemania, Inglaterra, Países Bajos y Escandinavia, se encontró pronto bajo predominio de los monarcas y de la Iglesia. La consecuencia de esto fue la supremacía de la medicina sobre la cirugía y de la teología cristiana sobre las ciencias de la naturaleza ${ }^{10}$. 
Durante la época de las invasiones germánicas se perdió gran parte del legado quirúrgico romano y griego, por ignorancia y un largo desuso. Los cristianos prácticamente habían abandonado los procedimientos quirúrgicos complejos, dejando la curación en manos del Espíritu Santo ${ }^{9,10}$. Con el tiempo se crearon dos tipos de cirujanos: el de toga larga, que había estudiado en latín, llamado por esto latinista; el de toga corta, cirujano-barbero, que por no saber latín se llamaba romancista.

Mientras los médicos, a menudo judíos doctos en medicina árabe, atendían a los nobles, alto clero y ricos burgueses, el pueblo se veía obligado a confiar en curanderos, barberos y flebótomos. Por otro lado, la anatomía basada en la disección de cadáveres humanos, largamente prohibida, comenzó lentamente a entrar en vigencia, al principio con fines legales y luego para la búsqueda de las causas de enfermedad. Con el desarrollo de la anatomía vino el de la cirugía, no considerada ya como una profesión indigna del sabio, sino como una parte inherente del arte de curar.

\section{Conclusiones}

La dietesis era una forma de salud total para el ciudadano y la polis griegos.

Platón es el fundador de la dualidad metafísica. La separación de enseñanza y práctica entre médicos y cirujanos se debe a la noción de separación entre cuerpo y alma.

El predominio de los monarcas y de la Iglesia medievales trajo como consecuencia la supremacía de la medicina sobre la cirugía y de la teología cristiana sobre las ciencias de la naturaleza.

La estratificación en clases de los estados medievales fue el sustrato para el médico filósofo, el cirujano práctico y el iletrado, el equipo de salud del renacimiento y la reforma.

\section{Referencias}

1. Colli G. Filósofos sobrehumanos. Biblioteca de Ensayo. Ed. Siruela. Barcelona, 2011.

2. Colli G. El nacimiento de la filosofía. Tusquets Editores. Buenos aires. 2010.

3. Jaeger W. Paideia. Los ideales de la cultura griega. Fondo de Cultura Económica. México. 2008.

4. Fierro Correa G. Aportes para un estudio de la formación de la idea científica de la naturaleza en los primeros filósofos griegos. En http://cabierta.uchile.cl/revista/31/ index.php [Consultado el 23 de noviembre de 2011].

5. Hipócrates. Sobre la enfermedad sagrada. Tratados. Biblioteca Gredos. Madrid, 1982, Barcelona, 2007.

6. Hipócrates. Sobre las aguas, aires y lugares. Ibid.

7. Aristóteles. Física. Trad. G. R. de Echandía. Ed. Gredos. Madrid. 1995.

8. Hipócrates. Sobre la dieta. Tratados. Biblioteca Gredos. Madrid, 1982, Barcelona, 2007.

9. Lyons AS, Petrucelli JR. Historia de la medicina. Edic. Doyma. Barcelona. 1984.

10. Laín Entralgo P. Historia de la medicina. Salvat Editores. Barcelona. 1978.

11. Platón. Fedro. Biblioteca Clásica Gredos. Barcelona. 2008.

12. Platón. Fedón. Edición de Francisco L. Lisi; traducción de Luis Gil Fernández. Madrid: Tecnos, 2002.

13. Nietzsche F. El Crepúsculo de los Ídolos. Traducción de A. Sánchez Pascual. Ed. Alianza. Madrid. 1979.

14. Platón. La República. Ediciones Unam. México 1959.

15. Nietzsche F. Voluntad de Poder. Edaf. Madrid. 1994.

16. Ferrater Mora J. Diccionario de Filosofía. Editorial Sudamericana. Buenos Aires. 1971.

17. Hipócrates. Sobre la medicina antigua. Tratados. Biblioteca Gredos. Madrid, 1982, Barcelona, 2007.

18. Maimónides. Obras Médicas. Ed. El Almendro. Granada. 2009.

19. Rhazes. La medicina árabe: el clínico Rhazes. Briceño Iragorry L. Ed. Ateproca. Caracas. 2008. 